\title{
Signal Association Approach Based on Doppler frequency Shift for Multi-Passive-Heterogeneous-Sensor System
}

\author{
Hairui Pan ${ }^{1, a}$, Yourong Lu ${ }^{1, b}$, Qian ${ }^{1, c}$ Cai and GangXu ${ }^{1, d}$ \\ ${ }^{1}$ Southwest Electronics and Telecommunication Technology Research Institute,Chendu,610041, \\ China;

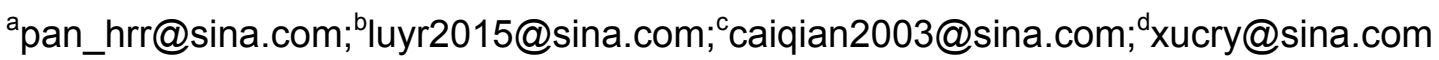

Keywords: Doppler frequency, signal association, wavelet ridge, STFT.

\begin{abstract}
This paper presents a signal association algorithm based on the Doppler frequency for the problem of signals inter-associated among multi-passive-heterogeneous-sensor system, radar and ultra-short wave signals are the specifically associated objects. This paper analyses the necessary and sufficient condition of signal association, by deeply analyzing, we figures out the factors and specific association probability expression. Simulation results show that method of association is effective and the associated probability maybe high under certain condition.
\end{abstract}

\section{Introduction}

Compared to single passive sensor, multi-passive- heterogeneous-sensor system can provide more information in the dimension of time, space, property, etc. In order to further improving the network of signal sensor system under big data background, analyzing relationships between signals become a hot topic. Signal association among multi-passive- heterogeneous-sensor system is intended to clarify whether the relationship and what kind of relationship exists among the mass signals. Currently, data association--academic research focuses on the correspondence between data and targets[1-6], but research about relationship between signals is few. That is because of the development of network of sensors is not so flourishing as today, on the other hand is that Signal association among multi-passive-heterogeneous-sensor system is difficult and system involves numerous species. Radar and ultra-short wave signals are the specific research objects in this paper. We carry on research about signals association from the frequency domain, which recognizes the subset of signal who are from the same target.

\section{Signal Association Principle}

Assuming that radar and ultra-short wave signals exist in a same certain period while the target is flying, sensors are located at the same place, the scene is shown in Fig.1.

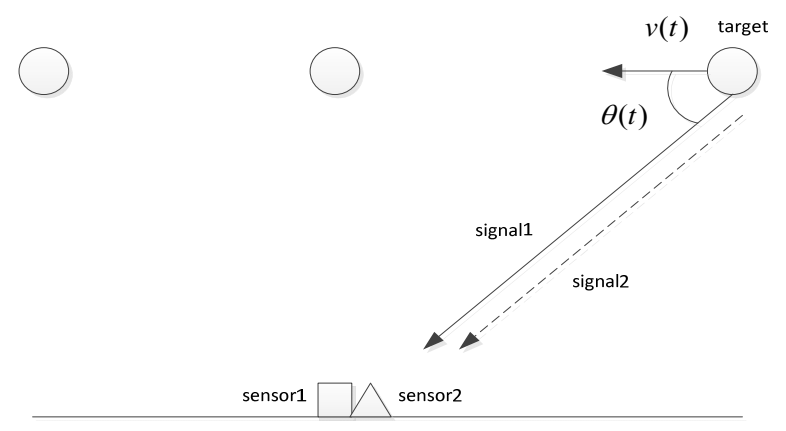

Fig. 1.scene of target and sensors

Doppler formula is as follows: 


$$
f_{d}(t)=\frac{f_{c}}{c} v(t) \cos \theta(t)
$$

Where $f_{d}(t)$ is Doppler frequency, $f_{c}$ is signal carrier frequency, $c$ is the speed of light, $v(t)$ is the speed of the target, $\theta(t)$ represents the angle between direction of speed and ligature from target to sensor.

Supposing that radar signal and ultra-short wave signal are from the same target, their carrier frequency are $f_{d, e_{1}}(t)$ and $f_{d, e_{2}}(t)$ respectively, then their relationship can be described as:

$$
\frac{f_{d, e_{1}}(t)}{f_{d, e_{2}}(t)}=\frac{f_{c 1} \cdot v(t) \cdot \cos \theta(t)}{f_{c 2} \cdot v(t) \cdot \cos \theta(t)}=\frac{f_{c 1}}{f_{c 2}}=\text { const }
$$

Studying in the period $\left[t_{0}, t_{0}+T\right]$ ( $T$ is small amount), signals' membership of target will not change, then signal $e_{1}(t)$ and $e_{2}(t)$ are associated or not associated. The following analysis show that Doppler frequency of signals satisfy (2), but the signals are not associated. Sensor coordinates can be set at the position $(0,0)$. At the time of $t_{0}$, coordinate of target which signal $e_{1}(t)$ belongs to can be set at the position $\left(x_{1}, y_{1}\right)$, coordinate of target which signal $e_{2}(t)$ belongs to can be set at the position $\left(x_{2}, y_{2}\right)$. Due to $T$ is a small value, targets can be seen as uniform linear motion, their speeds are $\left(v_{1, x}, v_{1, x}\right)$ and $\left(v_{2, x}, v_{2, x}\right)$ respectively, and $\forall t \in\left[t_{0}, t_{0}+T\right](2)$ are always true, after some derivation and simplification, targets' parameters obtained satisfy (3) ,namely

$$
\left\{\begin{array} { c } 
{ x _ { 1 } ^ { 2 } + y _ { 1 } ^ { 2 } = x _ { 2 } ^ { 2 } + y _ { 2 } ^ { 2 } } \\
{ v _ { 1 , x } ^ { 2 } + v _ { 1 , y } ^ { 2 } = v _ { 2 , x } ^ { 2 } + v _ { 2 , y } ^ { 2 } } \\
{ x _ { 1 } v _ { 1 , x } + y _ { 1 } v _ { 1 , y } = x _ { 2 } v _ { 2 , x } + y _ { 2 } v _ { 2 , y } }
\end{array} \text { or } \quad \left\{\begin{array}{c}
v_{1, x}^{2}+v_{1, y}^{2}=v_{2, x}^{2}+v_{2, y}^{2} \\
y_{1} v_{1, x}-x_{1} v_{1, y}=0 \\
y_{2} v_{2, x}-x_{2} v_{2, y}=0
\end{array}\right.\right.
$$

Let event A: signal $e_{1}(t)$ and $e_{2}(t)$ are associated; event B: signal $e_{1}(t)$ and $e_{2}(t)$ are not associated (namely they are from two targets); event $\mathrm{C}: \forall t \in\left[t_{0}, t_{0}+T\right]$, (2) are always true. Then

$$
P(A \mid C)+P(B \mid C)=1
$$

Assuming that the number of target is $N$, the distance between sensor and all targets are independent and identically distribution, $d \sim U\left[d_{1}, d_{2}\right]$ unit: $\mathrm{km}$; the speeds of all targets areindependent and identically distribution, $v \sim U\left[v_{1}, v_{2}\right], \mathrm{cm} / \mathrm{s}$; the directions of all speeds (relative to the $\mathrm{X}$ axis) are independent and identically distribution, $\psi \sim U\left[\psi_{1}, \psi_{2}\right]$, unit: degree; the angles between direction of speed and ligature from corresponding target to sensor are independent and identically distribution, $\theta \sim U\left[\theta_{1}, \theta_{2}\right]$ unit is degree. Under above assumptions, obviously, when $\forall t \in\left[t_{0}, t_{0}+T\right](2)$ are true, then the association probability of signal $e_{1}(t)$ and $e_{2}(t)$ is $100 \%$.

In fact, due to noise and other factors, (2) is difficult to guarantee identity. (5) is actual judgment, $\hat{f}_{d, e_{1}}(t)$ and $\hat{f}_{d, e_{2}}(t)$ are estimation of Doppler frequency.

$$
\left|\frac{\hat{f}_{d, e_{1}}(t)}{\hat{f}_{d, e_{2}}(t)}-\frac{f_{c 1}}{f_{c 2}}\right| \leq \Delta \frac{f_{c 1}}{f_{c 2}}
$$

The basic idea to calculate the association probability is to seek the range of parameters when (5) is true, $\forall t \in\left[t_{0}, t_{0}+T\right]$ Let $v_{1}^{\prime}=\sqrt{v_{1, x}^{2}+v_{1, y}^{2}}, v_{2}^{\prime}=\sqrt{v_{2, x}^{2}+v_{2, y}^{2}}$, the angles between direction of speed and ligature from target to sensor are $\theta_{1}^{\prime}(t), \theta_{2}^{\prime}(t)$. The maximum values of $\theta_{1}^{\prime}(t)$ and $\theta_{2}^{\prime}(t)$ are $\Delta \theta_{1}^{\prime}, \Delta \theta_{2}^{\prime}$, respectively. Due to $\Delta$ is small, solutions of (5) just lead to (3) some small perturbations. After some simplification, $v_{1}^{\prime}, v_{2}^{\prime}$ and $\theta_{1}^{\prime}(t), \theta_{2}^{\prime}(t)$ satisfy (6). 


$$
\left\{\begin{array}{c}
v_{2}^{\prime}(1-\Delta) \leq v_{1}^{\prime} \leq v_{2}^{\prime}(1+\Delta) \\
\theta_{1}^{\prime}(t) \in\left[-\Delta \theta_{1}^{\prime}, \Delta \theta_{1}^{\prime}\right] \\
\theta_{2}^{\prime}(t) \in\left[-\Delta \theta_{2}^{\prime}, \Delta \theta_{2}^{\prime}\right]
\end{array}\right.
$$

By (4), association probability of signal $e_{1}(t)$ and $e_{2}(t)$ is:

$$
P(A \mid C)=1-C_{N}^{2}\left\{\left[1-\frac{\left(v_{2}-v_{1}-v_{2}^{\prime} \Delta\right)^{2}}{\left(v_{2}-v_{1}\right)^{2}}\right] \times\left[\frac{\left(2 \Delta \theta_{1}^{\prime}\right) \times\left(2 \Delta \theta_{2}^{\prime}\right)}{\left(\theta_{1}-\theta_{2}\right)^{2}}\right]\right\}
$$

\section{Signal Association Model}

Specific method of signal association is shown in Fig .2.

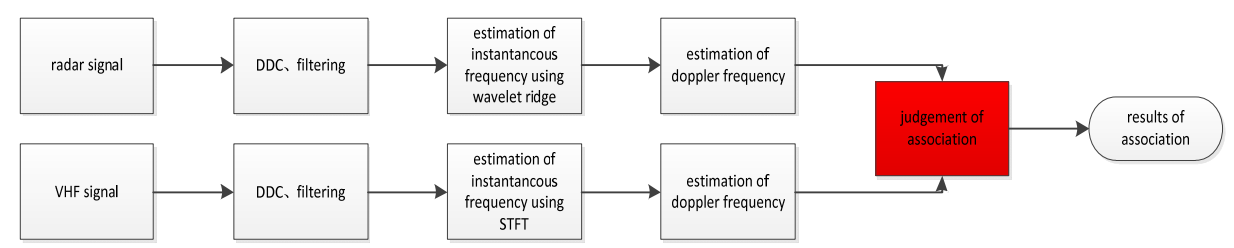

Fig. 2. method model of signal association

\section{Doppler frequency estimation of radar signal}

Assuming that target emits coherent phase pulse signals and its mathematical model can be expressed as:

$$
e_{1}(t)=\sum_{n=-\infty}^{\infty} \operatorname{rect}\left(\frac{t-n T}{\tau}\right) \cdot \cos \left(2 \pi f_{c 1} t+\varphi_{1}\right)
$$

where $\operatorname{rect}\left(\frac{t}{\tau}\right)=\left\{\begin{array}{c}1,0 \leq t<\tau \\ 0, \text { others }\end{array}, f_{c 1}\right.$ is carrier frequency, $\varphi_{1}$ is initial phase.

The received signal after spectrum moved to zero-IF is

$$
r_{1}(t)=\sum_{n=-\infty}^{+\infty} \operatorname{rect}\left(\frac{t-n T}{\tau}\right) \cdot \cos \left[2 \pi \int f_{d, e_{1}}(t) d t+\varphi_{1}\right]
$$

We use wavelet ridge method in [4-5] to seek instantaneous frequency $f_{d, e_{1}}(t)$.

\section{Doppler frequency estimation of ultra-shortwave signal}

Mathematical model of ultra-short wave signal is:

$$
e_{2}(t)=s(t) \cdot \exp \left\{j\left(2 \pi f_{c 2} t+\varphi_{2}\right)\right\}
$$

Where $f_{c 2}$ is a carrier frequency in VHF, $s(t)$ is baseband signal of MPSK, $\varphi_{2}$ is initial phase.

Considering the Doppler frequency shift, after signal changed to a small intermediate frequency , the received signal model can be expressed as:

$$
r_{2}(t)=\sum_{n=-\infty}^{+\infty} s(t) \cdot \exp \left\{j\left[2 \pi f_{I} t+2 \pi \int f_{d, e_{2}}(t) d t+\varphi_{2}\right]\right\}
$$

Before STFT transformation of $r_{2}(t)$, we must reduce the affect from modulation by M-power . After STFT, searching for maximum in local spectrum ,then it's easy to calculate Doppler frequency $f_{d, e_{2}}(t)$.

\section{Simulation}

\section{Verifying estimated Doppler frequency}

The initial position of target is $(1000,1000)$, initial velocity is $(-200,0)$, time of simulation is 30s. The radar signal's parameters are as follows: carrier frequency $f_{c 1}$ is $1096 \mathrm{MHz}$, pulse frequency $\mathrm{T}$ is 
$10 \mathrm{kHz}, \mathrm{SNR}=10 \mathrm{~dB}$, sample rate $f_{s 1}$ is $2000 \mathrm{~Hz}$. The ultra-short wave signal's parameters are as follows: modulation scheme is QPSK, carrier frequency $f_{c 2}$ is $365.333 \mathrm{MHz}, \mathrm{SNR}=5 \mathrm{~dB}$, small intermediate frequency $f_{I}$ is $5 \mathrm{kHz}$, over-sample rate is 32 , sample rate $f_{\mathrm{s} 2}$ is $32 \mathrm{kHz}$, window function of short-time Fourier transform is rectangular and its length is 32000 ,namely the resolution of time is $1 \mathrm{~s}$.

Fig.3(a) is the theoretical and estimated value about Doppler frequency of radar signal, root mean square error RMSE $=0.1583$, the estimation result is high precision and ideal; Fig.3(b) is the theoretical and estimated value about Doppler frequency of ultra-short wave signal, root mean square error $\mathrm{RMSE}=0.053$, the estimation result seems to be good. After estimating, we can get the ratio of Doppler frequency, according to (5) we can calculate $\Delta \min =0.0038$.

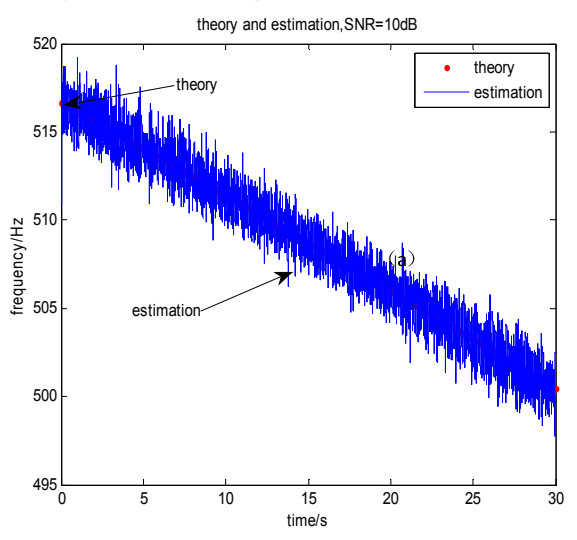

(a)

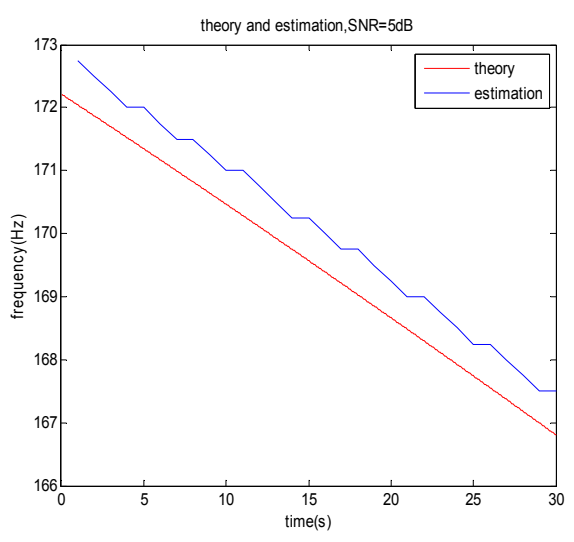

(b)

\section{Analysis of association results}

Fig. 3. theory and estimation of Doppler frequency

Assuming that the minimum speed of target is $100 \mathrm{~m} / \mathrm{s}$, the maximum speed is $500 \mathrm{~m} / \mathrm{s}$, that is $v_{1}=100, v_{2}=500$; the angle between direction of speed and ligature from target to sensor is $0^{\circ}$, the maximum value is $360^{\circ}$; namely, $\theta_{1}=0^{\circ}, \theta_{2}=360^{\circ}$; $v_{2}^{\prime}$ is the speed of any one of the two targets'.

As can be seen from Fig.4, when a certain number of targets is, the association probability decreases with the target speed increasing and increases with $\Delta$ decreasing. When the number of targets is less than 300 , the speed of target is target is not more than $300 \mathrm{~m} / \mathrm{s}$, the signal association probability is above $92 \%$.

\section{Summary}

Under the background of big data, the signal association among multi-passive-heterogeneoussensorsystem takes more and more people's attention .Lots of literatures studying this problem are from the view of airspace. This paper researches the relationship that radar and ultra-shortwave signal belong to the same target, then we first propose a criterion by using Doppler frequency. The analysis show that association probability of this method is $100 \%$ in theory. Considering the measurement error of Doppler frequency under real circumstance, we make some theoretical derivation and give the expression of association probability. Simulation results show that associated probability of proposed method is high. 


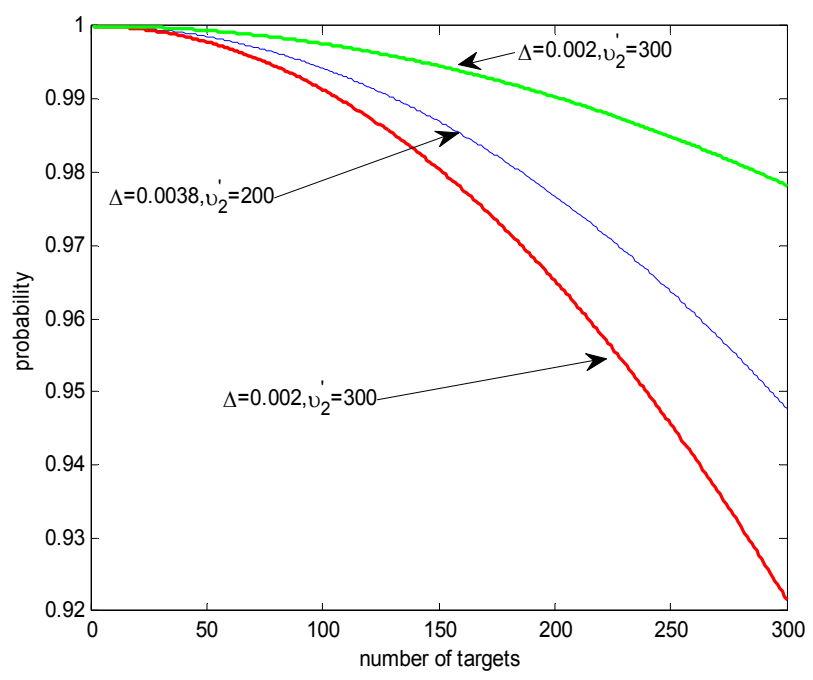

Fig. 4. association probablility of $e_{1}(t)$ and $e_{2}(t)$

\section{References}

[1] Singer R A,Sea R G.A new filter for optimal tracking in dense multi-target environment. In:Proceeding of the ninth Allerton Conference Circuit and System Theory.201-211(1971)

[2] Jitendra R. Raol, Multi-Sensor Data Fusion with Matlab,CRC Press, Taylor \& Francis Group, ISBN 978-1-4398-0003-4(2010)

[3] Ren C. Luo, Ying Chih Chou and Ogst Chen, Multi-sensor Fusion and Integration: Algorithms, Applications and Future Research Directions,Proceedings of the 2007 IEEE International Conference on Mechatronics and Automation, Harbin, China(2007)

[4] Ashraf M. Aziz. Fuzzy track-to-track association and track fusion approach in distributed multi-sensor multi-target multiple-attribute environment. Signal Processing,VOL.87, No.6, pp. 1474-1492(2007)

[5] Han Chongzhao, Zhu Hongyan, Duan Zhansheng. Multi-source information fusion [M]. Tsinghua University Press (2006)

[6] H. Chen, T. Kirubarajan and Y. Bar-Shalom, Performance Limits of Track-to-Track Fusion vs.Centralized Estimation, IEEE Trans. Aerosp.Electronic Systems, 39(2):386-400 (2003)

[7] Mingqiu. Ren, Jinyan. Cai, Yuanqing. Zhu. Radar emitter signal classification based on mutual information and fuzzy support vector machines, Proc. IEEE. Int. Confon Signal processing, ICSP2008,volume 2, pp. 1642-1646,(2008)

[8] Fan Yang,Shenghua Dai.A single-chip solution for Railway frequency shift signal detection[J].IEEE WCNIS pp:197-201(2010)

[9] J. M. Aller, T. G. Habetler, R. G. Harley, R. M. Tallam, and S. B. Lee, Sensorless speed measurement of AC machines using analytic wavelet transform, IEEE Trans. Ind. Appl., vol. 38, no. 5, pp. 1344-1350 (2002)

[10] Peng Geng,Huang Zhi-tao,JIANG Wen-li,ZHOU Yi-yu. Blind Doppler parameters estimation of satellite communication signals. Systems Engineering and Electronics. 32(4): 674-677(2010) 